\title{
Mariapanteles (Hymenoptera, Braconidae), a new genus of Neotropical microgastrine parasitoid wasp discovered through biodiversity inventory
}

\author{
James B. Whitfield ${ }^{1, \dagger}$, José L. Fernández-Triana ${ }^{2,3, \ddagger}$, Daniel H. Janzen ${ }^{4, \S}$, \\ Winnie Hallwachs ${ }^{4,1}$, M. Alex Smith ${ }^{2, \pi}$, Sophie Cardinal ${ }^{3, \#}$
}

I Department of Entomology, 320 Morrill Hall, 505 S. Goodwin Ave., University of Illinois, Urbana, IL 61801 USA 2 Department of Integrative Biology \& the Biodiversity Institute of Ontario, University of Guelph, Guelph, Ontario, N1G 2W1 CANADA 3 Canadian National Collection of Insects, Ottawa, ON K1A OC6, CANADA 4 Canadian National Collection of Insects, Ottawa, ON K1A OC6, CANADA

† urn:lsid:zoobank.org:author:7A98AB5F-552D-4437-8F5D-C593CA713506

† urn:lsid:zoobank.org:author:4469D91F-BBC1-4CBF-8263-EBFE2A95E4BF

§ urn:lsid:zoobank.org:author:4491369A-CFA6-4614-AC09-1137CCD06F9A

| urn:lsid:zoobank.org:author:68F37FFD-B6AB-49AD-A1AD-1C84B2FB94C9

I urn:lsid:zoobank.org:author:E46EE6EB-E096-4FCD-BF5A-F91D4A8294EE

\# urn:lsid:zoobank.org:author:9D43F9ED-9B25-4C55-A99E-84D62F7204CO

Corresponding author: James B. Whitfield (jwhitfie@life.illinois.edu)

Academic editor: Gavin Broad | Received 4 May 2012 | Accepted 29 June 2012 | Published 17 July 2012

urn:lsid:zoobank.org:pub:51CE38E7-5FB4-4D70-8574-7D27FD935233

Citation: Whitfield JB, Fernández-Triana JL, Janzen DH, Hallwachs W, Smith MA, Cardinal S (2012) Mariapanteles (Hymenoptera, Braconidae), a new genus of Neotropical microgastrine parasitoid wasp discovered through biodiversity inventory. ZooKeys 208: 61-80. doi: 10.3897/zookeys.208.3326

\begin{abstract}
A new genus of microgastrine parasitoid wasps, Mariapanteles Whitfield \& Fernández-Triana, gen. n., is described from rain forests of the Neotropics. The new genus is related to the common and speciose genus Pseudapanteles, but can be distinguished from the latter by having a complete transverse carina on the propodeum which forks around the spiracles. A molecular analysis based on data from COI from specimens of the proposed new genus plus possibly related genera confirms its generic distinctness. A key to two known species, $M$. felipei Whitfield, sp. n. (Costa Rica) and M. dapkeyae Fernández-Triana, sp. n. (Brazil) is provided. Evidence from collections suggests that there are other undescribed Neotropical congenerics. Specimens of Mariapanteles were likely confused in the past with the genus Beyarslania (referred to as Xenogaster until recently) but present information suggests that Beyarslania is restricted to the Afrotropical region while the Neotropical species clearly belong to a different genus, which we propose as new.
\end{abstract}

Copyright James B. Whitfield et al. This is an open access article distributed under the terms of the Creative Commons Attribution License 3.0 (CC-BY), which permits unrestricted use, distribution, and reproduction in any medium, provided the original author and source are credited. 


\section{Keywords}

Microgastrinae, new genus, Area de Conservación Guanacaste, Costa Rica, Brazil, Neotropics, parasitoid wasp, rain forest

\section{Introduction}

In the past few decades, biodiversity inventories in the neotropics have begun to incorporate intensive rearing programs for caterpillars, their food plants, and their parasitoids. The most extensive of these programs is in the Area de Conservación de Guanacaste (ACG) in northwestern Costa Rica. ACG contains 130,000 terrestrial hectares of contiguous conserved dry forest, cloud forest, and rain forest, extending from the Pacific Ocean to 2,000 m elevation and then down into the Caribbean lowlands (Janzen 2000, Janzen and Hallwachs 2011). The caterpillar/plant/parasitoid inventory is one of the main components of ACG conservation (e.g. Janzen and Hallwachs 2011, 2012; Janzen et al. 2009; Smith et al. 2006, 2007, 2008; Sharkey et al. 2011; Burns et al. 2007, 2008; Schauff et al. 2001; Gauld and Janzen 2004). Microgastrinae have been one of the most intensively studied groups of ACG parasitoids (e.g., Valerio et al. 2009; Smith et al. 2008; Grinter et al. 2009; Deans et al. 2003, Whitfield et al. 2011), but many hundreds of species of these tiny wasps still remain to be discovered and described. Microgastrines are probably the single most speciose higher taxon of parasitoids of Lepidoptera (Whitfield 1995, 1997), and it is estimated that $800+$ species occur in ACG alone (Janzen and Hallwachs, inventory in progress).

The ACG inventory has encountered a rain forest species of Microgastrinae that superficially resembles the rare Old World genus Beyarslania - widely known as Xenogaster until a recent nomenclatural change (Kocak and Kemal 2009). This genus is only known from a single described species from South Africa, but undescribed species have been thought to exist in the Neotropics (Whitfield 1997; Campos and Diego 2001). As we studied the ACG specimens and other similar Neotropical specimens, we realized that these undescribed species are more informatively placed in a new genus, which we erect and describe here along with descriptions of two species contained within it. A molecular phylogenetic analysis based on data from the COI ("barcoding") gene is included to provide a further test of the monophyly of this putative new genus.

\section{Methods}

The specimens for this study came from two main sources: the ACG inventory (Janzen and Hallwachs 2012, Janzen et al. 2009) and unsorted Neotropical wasps from the Canadian National Collection of Insects (CNC) in Ottawa. The genotype for the new genus has been deposited in the Smithsonian Institution (NMNH) and the remaining specimens have been deposited in the CNC, Illinois Natural History Survey (INHS) and the Natural History Museum, London (BMNH). 
The morphological terms and morphological measurements follow mostly Wharton and Sharkey (1997) and Valerio and Whitfield (2009).

Photos were taken with a Keyence VHX-1000 Digital Microscope, using a lens with a range of 13-130x. Plates for the illustrations were prepared using Adobe Photoshop, but images were not digitally enhanced.

DNA barcodes for these and all other ACG inventory Microgastrinae were obtained using DNA extracts prepared from single legs using a glass fibre protocol (Ivanova et al. 2006). Extracts were re-suspended in $30 \mu \mathrm{l}$ of $\mathrm{dH} 2 \mathrm{O}$, and a 658-bp region near the 5' terminus of the COI gene was amplified using standard primers (LepF1-LepR1) following established protocols (Smith et al. 2006, 2007, 2008). If the initial 658 bp amplification was not successful composite sequences were generated using internal primers. Primer information for individuals sequences can be retrieved from the Barcode of Life Data System (BOLD) (Ratnasingham and Hebert 2007) using the accessions detailed in the online supplementary table , but primers are as detailed in Smith et al. (2008). Full details of methodology are as in (Smith et al. 2006, 2007, 2008). All sequence data are available on BOLD (www.barcodinglife. org) in the public Dataset, "Mariapanteles (Hymenoptera: Braconidae), a new genus of Neotropical microgastrine parasitoid wasp discovered through biodiversity inventory". All collection information, BOLD, and GenBank accessions for all sequences are listed in the Table in online supplementary materials.

All available DNA barcodes for Pseudapanteles (32 New World species, most of which are undescribed) and Mariapanteles (2 species) that were at least 300 bp long were downloaded from BOLD (see the online supplementary table). DNA barcodes for selected species of Prasmodon, Diolcogaster, Microplitis, Cotesia, Apanteles, and Neoclarkinella were also downloaded to be used as outgroups in the phylogenetic analyses. Genbank accession numbers of all sequences used are given in Table 1. Prior to analysis, identical Pseudapanteles sequences were removed from the dataset so that each unique sequence was only represented once. Sequences were aligned in Geneious Pro 5.5.6 (Drummond et al. 2011) using default settings for MUSCLE alignment. The first character was deleted from the aligned matrix because most sequences were missing this character. Bayesian phylogenetic analysis was performed in MrBayes v.3.1.2 (Ronquist and Huelsenbeck 2003) through the CIPRES Science Gateway V.3.1 (Miller et al. 2010). Model selection was based on Bayesian Information Criteria as implemented in JModelTest v.0.1.1 (Posada 2008). Two independent analyses with 4 chains each were run in parallel for 10 million generations under a $G T R+I+G$ model. The parameter trace files of each run were observed in Tracer v.1.5 (Rambaut and Drummond 2009) to verify that the runs had converged on the same stationary distribution, and to decide on the appropriate number of generations to discard as burn-in. A maximum clade credibility tree was constructed from these 18 million post-burn-in generations in TreeAnnotator v1.7.0 (Rambaut and Drummond 2012). The above protocol was followed for two additional analyses in which all $3^{\text {rd }}$ codon positions were removed from the dataset to correct for potential problems stemming from saturation in $3^{\text {rd }}$ codon positions of COI. 
Table I. Specimens included in the COI molecular analyses and their GenBank Accession numbers. Sample IDs are DHJPAR numbers (assigned to ACG specimens submitted to BOLD) or other IDs submitted to BOLD. More complete data on all specimens are included in Supplementary File 1.

\begin{tabular}{|c|c|c|}
\hline Taxon & Sample ID & Genbank Accession Number \\
\hline Apanteles Rodriguez03 & DHJPAR0012802 & JQ847281 \\
\hline Apanteles Rodriguez05 & DHJPAR0012285 & EU396474 \\
\hline Apanteles Rodriguez169 & DHJPAR0038032 & JQ848826 \\
\hline Cotesia Whitfield03 & DHJPAR0013374 & JQ848576 \\
\hline Diolcogaster Choi04 & DHJPAR0004153 & HQ549146 \\
\hline Mariapanteles dapkeyae F.-T. & CNCHYM 03387 & JQ849377 \\
\hline Mariapanteles felipei Whitfield & DHJPAR0025453 & HQ549955 \\
\hline Mariapanteles felipei Whitfield & DHJPAR0025443 & JN282317 \\
\hline Microplitis Whitfield19 & DHJPAR0031685 & HQ548870 \\
\hline Neoclarkinella sp.jft10 & WAM 0011 & JQ852287 \\
\hline Neoclarkinella sp.jft11 & GOU 0608 & JQ849914 \\
\hline Prasmodon Whitfield02 & DHJPAR0038222 & HQ548705 \\
\hline Prasmodon Whitfield05 & DHJPAR0012956 & HQ548880 \\
\hline Pseudapanteles gouleti F.-T. & CAM 0874 & JQ848150 \\
\hline Pseudapanteles sp. jft8 & CNCHYM 03312 & JQ849707 \\
\hline Pseudapanteles sp. jft16 & CNCHYM 03343 & JQ575645 \\
\hline Pseudapanteles sp. jft19 & CNCHYM 03355 & JQ850586 \\
\hline Pseudapanteles sp. jft 23 & CNCHYM 03372 & JQ850275 \\
\hline Pseudapanteles sp. jft23 & CNCHYM 03372 & JQ850275 \\
\hline Pseudapanteles sp. jft 23 & CNCHYM 03369 & JQ854346 \\
\hline Pseudapanteles sp. jft25 & CNCHYM 03377 & JQ853286 \\
\hline Pseudapanteles sp. jft25 & CNCHYM 03377 & JQ853286 \\
\hline Pseudapanteles sp.jft29 & 10BBHYM-1279 & JQ852886 \\
\hline Pseudapanteles sp.jft29 & Micro0094 & JQ852261 \\
\hline Pseudapanteles sp.jft29 & Micro0269 & JQ850735 \\
\hline Pseudapanteles Whitfield01 & DHJPAR0004755 & JQ849938 \\
\hline Pseudapanteles Whitfield02 & DHJPAR0025345 & JQ850204 \\
\hline Pseudapanteles Whitfield05 & DHJPAR0031341 & JQ852695 \\
\hline Pseudapanteles Whitfield05 & DHJPAR0026205 & HQ549736 \\
\hline Pseudapanteles Whitfield05 & DHJPAR0033906 & JQ576585 \\
\hline Pseudapanteles Whitfield06 & DHJPAR0031347 & HQ930249 \\
\hline Pseudapanteles Whitfield06 & DHJPAR0031191 & JQ849350 \\
\hline Pseudapanteles Whitfield06 & DHJPAR0034081 & JQ853689 \\
\hline Pseudapanteles Whitfield07 & DHJPAR0013217 & JQ852406 \\
\hline Pseudapanteles Whitfield08 & DHJPAR0026281 & JQ849770 \\
\hline Pseudapanteles Whitfield09 & DHJPAR0027627 & JN281617 \\
\hline Pseudapanteles Whitfield09 & DHJPAR0027692 & HM430666 \\
\hline Pseudapanteles Whitfield09 & DHJPAR0027392 & JQ849832 \\
\hline Pseudapanteles Whitfield09 & DHJPAR0026088 & JQ854468 \\
\hline Pseudapanteles Whitfield09 & DHJPAR0026012 & JQ853711 \\
\hline Pseudapanteles Whitfield09 & DHJPAR0026026 & HQ549676 \\
\hline Pseudapanteles Whitfield09 & DHJPAR0027661 & JQ576067 \\
\hline Pseudapanteles Whitfield09 & DHJPAR0033842 & HQ550374 \\
\hline Pseudapanteles Whitfield09 & DHJPAR0031297 & JQ855464 \\
\hline Pseudapanteles Whitfield10 & DHJPAR0012880 & JQ854478 \\
\hline
\end{tabular}




\begin{tabular}{|c|c|c|}
\hline $\begin{array}{c}\text { Taxon } \\
\end{array}$ & Sample ID & Genbank Accession Number \\
\hline Pseudapanteles Whitfield11 & DHJPAR0031742 & JQ847036 \\
\hline Pseudapanteles Whitfield12 & DHJPAR0025380 & HQ550092 \\
\hline Pseudapanteles Whitfield14 & DHJPAR0025854 & HQ549888 \\
\hline Pseudapanteles Whitfield15 & DHJPAR0025751 & JQ849472 \\
\hline Pseudapanteles Whitfield17 & DHJPAR0026060 & JN281784 \\
\hline Pseudapanteles Whitfield18 & DHJPAR0027669 & JQ853458 \\
\hline Pseudapanteles Whitfield19 & DHJPAR0027440 & JQ848147 \\
\hline Pseudapanteles Whitfield19 & DHJPAR0026033 & JQ847890 \\
\hline Pseudapanteles Whitfield19 & DHJPAR0026268 & JQ852987 \\
\hline Pseudapanteles Whitfield19 & DHJPAR0027440 & JQ848147 \\
\hline Pseudapanteles Whitfield19 & DHJPAR0026033 & JQ847890 \\
\hline Pseudapanteles Whitfield19 & DHJPAR0026268 & JQ852987 \\
\hline Pseudapanteles Whitfield19 & DHJPAR0025022 & HQ926353 \\
\hline Pseudapanteles Whitfield19 & DHJPAR0026008 & JQ850045 \\
\hline Pseudapanteles Whitfield19 & DHJPAR0025959 & JQ849085 \\
\hline Pseudapanteles Whitfield19 & DHJPAR0025866 & JQ852050 \\
\hline Pseudapanteles Whitfield19 & DHJPAR0026534 & HQ550096 \\
\hline Pseudapanteles Whitfield19 & DHJPAR0027150 & HQ548886 \\
\hline Pseudapanteles Whitfield19 & DHJPAR0025831 & JQ574919 \\
\hline Pseudapanteles Whitfield20 & DHJPAR0040493 & JQ849256 \\
\hline Pseudapanteles Whitfield20 & DHJPAR0027329 & JQ848647 \\
\hline Pseudapanteles Whitfield20 & DHJPAR0039680 & JQ848927 \\
\hline Pseudapanteles Whitfield20 & DHJPAR0041914 & JQ847496 \\
\hline Pseudapanteles Whitfield21 & DHJPAR0038402 & EU396445 \\
\hline Pseudapanteles Whitfield21 & DHJPAR0027221 & JN282176 \\
\hline Pseudapanteles Whitfield23 & DHJPAR0031749 & JQ852705 \\
\hline Pseudapanteles Whitfield27 & DHJPAR0043058 & JQ850352 \\
\hline
\end{tabular}

\section{Results}

\section{Molecular confirmation of Mariapanteles monophyly}

Evidence (beyond the morphologcial data presented below) for the separation of these two genera comes from DNA barcode data (Figs 13, 14). When comparing the two species of Mariapanteles to 32 species of Pseudapanteles (most of them undescribed) for which there are CO1 sequences available in BOLD (www.boldsystems.com), the two genera are recovered as separate groups.

Bayesian analysis of the COI sequences, with and without $3^{\text {rd }}$ codon positions included, clearly supports Mariapanteles as being a separate distinct group from Pseudapanteles (Figs 13, 14). Pseudapanteles was recovered as being monophyletic with posterior probability (PP) of 1 when $3^{\text {rd }}$ codon positions were included and PP of 0.8 when $3^{\text {rd }}$ codon positions were removed. Monophyly of Mariapanteles was recovered with PP of 1 in both analyses. Furthermore, Mariapanteles was not recovered as the sister group to Pseudapanteles in either analysis. Several other genera included as potentially disrupting Mariapanteles monophyly also failed to do so in the analyses, so we have concluded that Mariapanteles is indeed a distinct genus. 


\section{Mariapanteles Whitfield \& Fernández-Triana, gen. n.}

urn:Isid:zoobank.org:act:917FB3C2-D102-4884-9FC4-787CC81AD5E9

http://species-id.net/wiki/Mariapanteles

Figs $1-12$

Type species. Mariapanteles felipei Whitfield sp. n., by present designation.

Genus diagnosis. Propodeum with a complete transverse carina that forks around spiracles and reaches the lateral margin of propodeum, where it intersects a raised lateral carina. Fore wing without areolet (veins $\mathrm{r}-\mathrm{m}$ and $3 \mathrm{RS}$ absent). First mediotergite with a sharp median groove on the basal half. The only other genera of Neotropical microgastrines with a complete transverse carina on the propodeum, Clarkinella and Prasmodon, both lack a medial groove on the first mediotergite and have an areolet in the forewing (a small areolet in Clarkinella, a large and quadrangular one in Prasmodon). Mariapanteles resembles Pseudapanteles in fore wing venation, shape of mediotergites 1 and 2, and general appearance of the body. However, Pseudapanteles has an elongate, bifurcate glossa, lacks a complete transverse carina on the propodeum, and the hypopygium has a large translucent fold with many pleats; the glossa of Mariapanteles is not bifurcate and the hypopygium has a median translucent fold with no or only a few pleats visible.

Description. Body length 2.4-2.6 mm, fore wing length $2.6-2.9 \mathrm{~mm}$, antenna about the same length as body. Pronotum with two lateral grooves present, the lower one excavated. Mesoscutum more or less uniformly sculptured by impressed punctures. Mesoscutum 1.3-1.4x wider than long. Mesoscutum and scutellum uniformly covered by dense, pale yellowish pilosity. Scutellum length/width at base 1.0-1.1X. Scutellar suture broad, with 4-8 costulae. Posterior band of scutellum polished. Scutellar lateral face with the polished area thin (15-25\% the face height) and about half the face width. Mesopleuron mostly smooth and glabrous, except for punctures on the anterior margin and setae on all margins. Metapleuron mostly smooth, with some punctures and setae in the apical half; metapleuron with a crenulate, longitudinal sulcus running from lower margin near metacoxa through spiracle. Metapleural carina raised, with a short lamella. Propodeum mostly smooth; median carina well defined and raised its entire length, and with a clearly complete transverse carina that reaches the spiracles and forks around them (there may also be additional, shorter transverse carinae, some of them radiating from the median carina but not reaching the spiracles). Transverse carina on propodeum delimiting two areas, the anterior, basal one being more or less horizontal; the posterior, apical one is declivous. Mediotergite 1 mostly smooth and with a deep medial groove on its basal half; slightly widening for the first quarter of its length, then narrowing towards apex. Mediotergite 2 mostly smooth, transverse, subtriangular to trapezoidal in shape. Mediotergite 3 and following, unsculptured, polished and with sparse setae. Hypopygium mostly inflexible but with a medial, translucent fold ventrally where none or few (1-2) pleats are distinguishable. Ovipositor sheaths fully setose, $0.7 \times$ as long as metatibia length. Metacoxa long, surpassing the length of the third metasomal tergite. Metatibial inner spur longer than outer spur, and 
about half the length of metatarsomere 1. Metafemur more than 3.0x as long as wide. Fore wing without an areolet, vein R1a longer than stigma length, and vein $\mathrm{r}$ and $2 \mathrm{RS}$ evenly curved to very slightly arched (with no clear limits between the two veins). Hind wing with edge of vannal lobe medially straight to slightly concave and with uniformly distributed setae that are shorter than those at base and apex of the lobe.

Distribution. The genus occurs in Central and South American rain forests. We describe two new species, one from Costa Rica (ACG, from rain forest at $400 \mathrm{~m}$ ) and one from Brazil (Mato Grosso and Goiás; the localities are presumed to have been rain forests at the time the specimens were collected). The CNC collection contains two additional specimens from other areas of Brazil that may represent additional species, but because they are singletons we do not describe them here. It is likely that more species of this new genus will be found in Neotropical rain forests.

Biology. Unknown. All specimens have been collected with Townes-type Malaise traps.

Etymology. Mariapanteles is dedicated to María Marta Chavarría Díaz of ACG and San Jose, Costa Rica, in recognition of her 30+ years of dedication to Costa Rican conservation, biodiversity systematics, and biodiversity development throughout Costa Rica, and very specifically within Area de Conservación de Guanacaste.

Comments. Mariapanteles is closely related to Pseudapanteles, and future revisions of the phylogeny of Microgastrinae might find that its erection renders Pseudapanteles paraphyletic. For example, and according to Mason (1981), some species of Pseudapanteles could have a multiple or indefinite transverse carina, in which case the complete transverse carina in Mariapanteles might be seen as the extreme in a continuum from having no transverse carina to having the complete transverse carina of Mariapanteles. However, we consider that the presence of a complete transverse carina on the propodeum, forking around the propodeal spiracles, may be a strong autapomorphy that defines Mariapanteles. There are only four other genera of Microgastrinae with a similar, complete transverse carina on the propodeum: Beyarslania, Clarkinella, Neoclarkinella, and Prasmodon. However, they all appear to be only distantly related to Mariapanteles because they all lack a sharp median groove on mediotergite 1 and/or have an areolet in the fore wing.

The described species of Pseudapanteles never have a complete transverse carina. Most of the specimens in collections just have a simple median carina, with only few species having irregular transverse striations arising along the length of the median carina (but even in those cases they never reach the spiracle and never form a fork around them). One example can be seen in the original description and pictures of the species Pseudapanteles gouleti Fernández-Triana, from Canada (Figure 18, page 24, in Fernández-Triana 2010). However, the carination pattern is not comparable to a complete transverse carina - as displayed by Mariapanteles.

The presence of a bifurcate glossa is a strong autapomorphy for Pseudapanteles (it is only present in three other distantly related Microgastrinae genera: Napamus, Promicrogaster and Sendaphne). Furthermore, the differences between Mariapanteles and Pseudapanteles with respect to the pleated area of the hypopygium are also consistent in the separation of these two genera. 
Currently Pseudapanteles has nine described species (Yu et al. 2009), and a wide distribution within the New World, ranging from Canada to South America (one of the species has also been introduced to Hawaii (Coulson 1992)). The actual number of species is much higher, and we have seen in collections several dozen undescribed species, mostly from the Neotropics. The vast majority of those specimens are remarkably invariant in having a bifurcate glossa and in lacking a transverse carina on the propodeum.

For all of the above reasons along with the molecular results, we have decided that Mariapanteles is a distinct, separate genus that may be closely related to Pseudapanteles.

As for the former records of Neotropical species of the genus Beyarslania (at that time called Xenogaster) (Whitfield 1997; Campos 2001), these are based on confusion with specimens of what we have described here as Mariapanteles. Based on the available evidence, we now consider Beyarslania to be restricted to the African tropics. The Neotropical specimens thought to belong to that genus should be identified as Mariapanteles.

\section{Key to Mariapanteles species described here}

$1 \quad$ Body colour mostly yellow in females and males —at most, males with tergite 3+ brown (Fig. 8); scutellum mostly smooth and scutellar suture with 4-6 costulae (Fig. 12); propodeum with a complete transverse carina that is clearly independent of other smaller transverse carinae (Figs 9, 12) [Mato Grosso and Goiás, Brazil] Mariapanteles dapkeyae sp. $\mathbf{n}$.

- $\quad$ Body colour in females mostly orange-yellowish but with some areas brown or reddish brown (apical edge of scutellum, metascutellum, some carina on propodeum, central area on mediotergites 3+) (Fig. 1), male with much darker coloration, especially in the interocellar area, mesosoma and metasoma (Fig. 2); scutellum with impressed punctures and scutellar suture with 6-8 costulae (Fig. 6); propodeum with a completely transverse carinae not always clearly delimited from other smaller transverse carina (Fig. 6) [ACG, northwestern Costa Rica]

Mariapanteles felipei sp. $\mathbf{n}$.

\section{Species descriptions}

Mariapanteles felipei Whitfield, sp. n. urn:lsid:zoobank.org:act:2A5E1320-5C93-45FE-AEB0-AF9382929A78 http://species-id.net/wiki/Mariapanteles_felipei

Figs 1-6

Holotype. Female (NMNH). COSTA RICA: Alajuela Province, Sector Rincon Rain Forest of ACG, Caribe, Rio Francia, 400 m, latitude 10.90093, longitude -85.28915; 11-17.vii.2007, Malaise Trap. Voucher code: DHJPAR0025453.

Paratype. Male (NMNH). Same data as for holotype, except for collecting date: 22-28.viii.2007. Voucher code: DHJPAR0025443. 
Description. Female. Antenna about the same length as body; body length 2.6 $\mathrm{mm}$; forewing $2.9 \mathrm{~mm}$. Head: face with shallow and sparse punctures and sparse, uniformly distributed setae; face width at antennal base/face width at clypeus edge: $1.1 \times$; intertentorial pit distance/face width at clypeus edge: $0.5 \times$; compound eye height/head height: $0.8 \times$; head height/width: $0.8 \times$; face width at antennal base/head maximum width: $0.6 \times$; malar space/basal width of mandible $1.3 \times$; clypeus width/height: $3.1 \times$. Length/width of flagellomeres: 2nd $(2.3 \times), 8$ th $(2.5 \times), 14$ th $(1.3 \times)$. Length of flagellomere $2^{\text {nd }} /$ length of flagellomere $14^{\text {th }}: 2.2 \times$. Ocello-ocular distance/posterior ocelli diameter: $2.3 \times$; distance between posterior ocelli/ocelli diameter: $1.4 \times$.

Mesosoma. Pronotum with two lateral grooves, the lower one excavated. Mesoscutum more or less uniformly sculptured by impressed punctures (distance between punctures about the same as their diameter). Mesoscutum 1.4× wider than long. Mesoscutum and scutellum uniformly covered by dense, pale-coloured pilosity. Scutellum similarly sculptured to mesoscutum. Scutellum length/width at base 1.0×. Scutellar suture broad, with 6-8 costulae. Posterior band of scutellum polished. Scutellar lateral face with polished area less than 30\% the face height and about half the face width. Mesopleuron mostly smooth and glabrous, except for punctures on the anterior margin and setae on the all margins; separated from metapleuron by a crenulated sulcus. Metapleuron mostly smooth, with some punctures and setae in the apical half; metapleuron with a crenulate, longitudinal sulcus running from lower margin near metacoxa through spiracle. Metapleural carina raised with a short lamella. Propodeum mostly smooth, with a median carina well defined and raised its entire length; and with a clearly complete transverse carina that reaches the spiracles and forks around them (also with additional, shorter transverse carinae, some of them radiating from the median carina but not reaching the spiracles). Transverse carina on propodeum delimiting two areas, the anterior, basal one being more or less horizontal, while the posterior, apical one is declivous.

Metasoma. Mediotergite 1 mostly smooth and with a deep medial groove over its basal half; slightly widening for the first quarter of its length, then narrowing towards apex; basal width/apical width 2.1x; length/apical width 4.8x. Mediotergite 2 mostly smooth, transverse, subtriangular to trapezoidal in shape; basal width/apical width $0.4 \times$; length/apical width $0.4 \times$. Mediotergite $31.5 \times$ the length of mediotergite 2. Mediotergite 3 and following unsculptured, polished and with sparse setae. Hypopygium mostly inflexible but with a median, translucid fold ventrally where no pleats are distinguishable. Ovipositor sheaths fully setose, $0.7 \times$ as long as metatibia length.

Legs. Metacoxa long, surpassing the length of the third metasomal tergum. Metatibial inner spur $1.6 \times$ the length of outer spur, and $0.6 \times$ the length of metatarsomere 1 . Metafemur $3.2 \times$ as long as wide.

Wings. Vein R1a $1.3 \times$ as long as stigma length. Stigma $3.1 \times$ as long as wide. Length of R1a about $12 \times$ as long as the distance between its end and the end of $3 \mathrm{RSb}$. Vein $r$ and 2RS evenly curved to very slightly arched, with no clear limits between the two veins. Vein $2 \mathrm{M}$ about the same length of vein $(\mathrm{RS}+\mathrm{M}) \mathrm{b}$. Edge of vannal lobe of hind wing medially straight to slightly concave and with uniformly distribute setae which are shorter than those at base and apex of the lobe. 

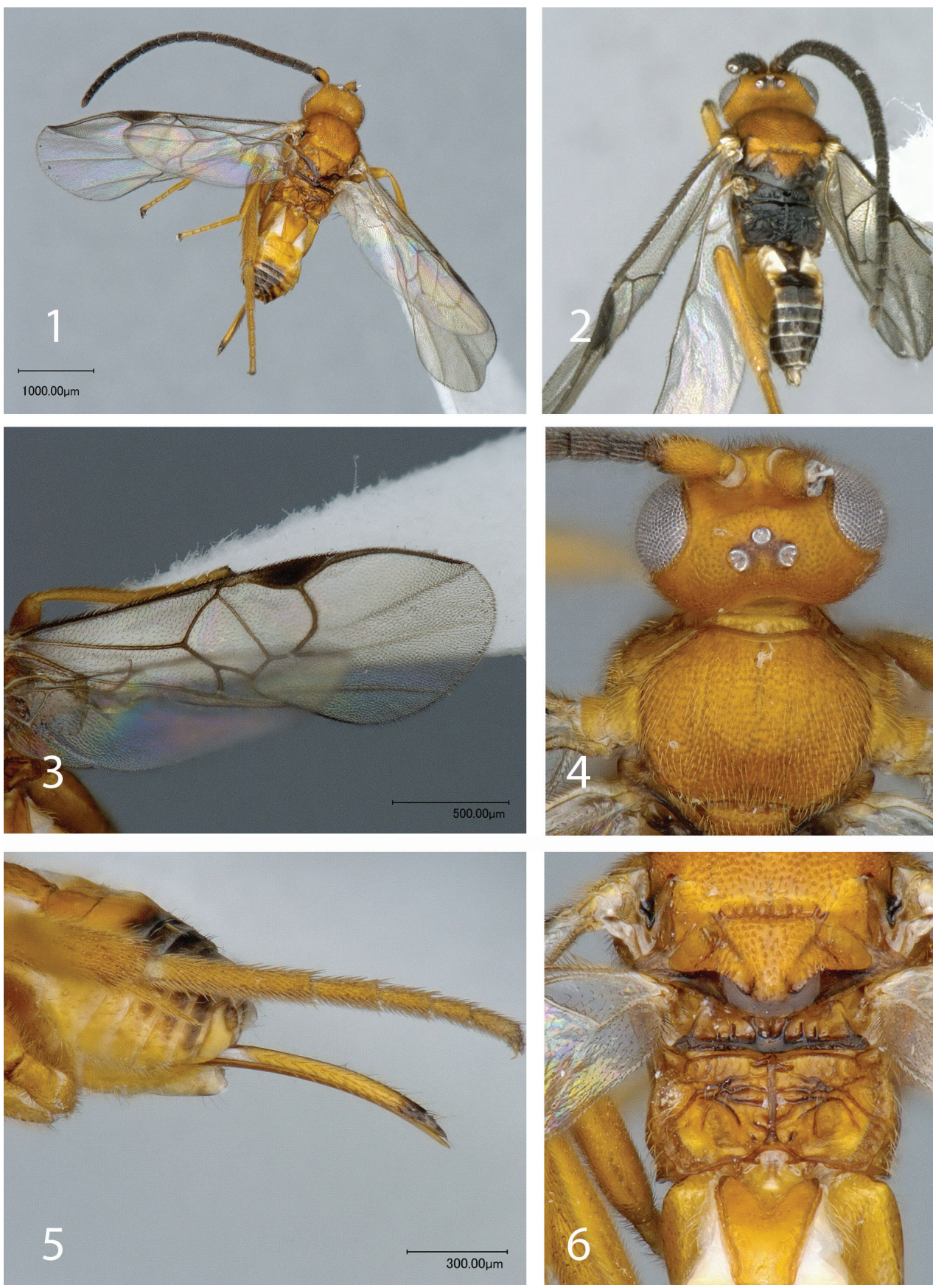

Figures I-6. Mariapanteles felipei Whitfield. I Dorsal habitus, female $\mathbf{2}$ Dorsal habitus, male $\mathbf{3}$ forewing, female $\mathbf{4}$ head and mesoscutum, dorsal view, female $\mathbf{5}$ hypopygium and ovipositor, lateral view 6 metanotum and propodeum, female, dorsal view. 
Colour: Mostly an orange-yellowish species. Antennal flagellomere and dorsal part of scape brown. Apical edge of scutellum, metascutellum and some carina on propodeum, reddish-brown. Central area on mediotergites 3 and following dark brown. Forewing stigma and most of the wing veins dark brown.

Male. Like the female except for darker coloration as follows: interocellar area, propodeum, metascutellum, apical edge of scutellum, most of the lateral face of scutellum, and most of mediotergites $2+$, dark brown to black.

Distribution. The known specimens were captured in July-August 2007 (full rainy season) by the same Malaise trap placed in old growth rain forest understory on the banks of Rio Francia, where it crosses the access road through Sector Rincon Rain Forest of ACG, at $400 \mathrm{~m}$.

Molecular data. The two known specimens bear the same DNA barcode. The nucleotide sequence in fasta format is:

$>$ Mariapanteles felipei

ATTTTATATTTTTTATTTGGAATATGATCTGGAATATTAGGATTTTCATTAAGAATAATTATCCGATTAGAGTTAGGCACACCAGGAAGATTAATTAGAAATGATCAAATCTATAATAGAATTGTTACATCACATGCTTTTATCATAATTTTTTTTATAGTTATACCAATTATAATTGGAGGATTTGGTAATTATTTAATTCCATTAATATTAGCAACTCCTGATATATCATTCCCACGAATAAATAATATGAGATTTTGATTACTAATTCCTTCATTATTTTTATTAATTTTTAGAAGATTTATTAATACAGGAGTAGGTACAGGTTGAACAGTTTATCCACCTTTATCATCAAATTTAGGACATAGAGG TATATCAGTTGATTTAGGAATCTTTTCTCTACATTTAGCAGGAGCCTCATCAATTATAGGAGCAATTAATTTTATTACAACAATTAAAAATATACGAGTTAAATTATTAAAAATAGATAAAATTTCTTTATTTACTTGATCAGTTTTAATTACAGCAATTTTATTATTATTATCTTTACCAGTTTTAGCAGGAGCAATTACTATACTTTTAACAGACCGAAATTTAAATACATCATTTTTTGATCCTTCAGGAGGTGGGGATCCAATTTTATACCAACATTTATTT

Etymology. Mariapanteles felipei is dedicated to Luis Felipe Chavarría Díaz of ACG and San Jose, Costa Rica, in recognition of his 30+ years of dedication to Costa Rican conservation, biodiversity systematics, and biodiversity development throughout Costa Rica, and very specifically within Area de Conservación de Guanacaste.

Comments. The biology of this species, collected with Malaise traps, is unknown. Since its inception in 1978, the ACG caterpillar and parasitoid inventory (Janzen et al. 2009) has achieved Microgastrinae rearings from 9,000+ wild-caught caterpillars and has Malaisetrapped 5,000+ individual Microgastrinae in dry forest, cloud forest and rain forest (Janzen and Hallwachs 2012, Smith et al. 2008); this intense effort has yielded only two conspecific individuals of Mariapanteles, both from the same Malaise trap a few weeks apart. While this may suggest that the species is "rare", it has been the experience of the ACG inventory that when the wasp is finally reared and therefore its host caterpillar known, or the Malaise trap is placed in the "right" place, it may well be found to be common. 


\section{Mariapanteles dapkeyae Fernández-Triana, sp. n.} urn:lsid:zoobank.org:act:EF2F8657-FEBB-4AEE-8501-06B104771B86 http://species-id.net/wiki/Mariapanteles_dapkeyae Figs $7-12$

Holotype. Female (CNC). BRAZIL: Mato Grosso, Sinop; x-xi.1975, Malaise Trap; M. Alvarenga col.

Paratype. 5 Females and 4 Males (CNC, with 1 female each deposited in INHS and BMNH). Same data as for holotype, except for collecting date (x.1974 for all specimens but two males with collecting date: x.1975). Two males deposited in the CNC have DNA Voucher codes: CNCHYM 03387 and CNCHYM 07145. 1 Female (CNC). BRAZIL: Goiás, Jatai; xi.1972; F. M. Oliveira col.

Description. Female. Antenna about the same length as body; body length 2.4 $\mathrm{mm}$; forewing $2.6 \mathrm{~mm}$. Head. Face with shallow and sparse punctures and sparse, uniformly distributed setae. Face width at antennal base/face width at clypeus edge: 1.1×; intertentorial pit distance/face width at clypeus edge: $0.4 \times$; compound eye height/head height: $0.8 \times$; head height/width: $0.8 \times$; face width at antennal base/head maximum width: $0.5 \times$; malar space/basal width of mandible $1.4 \times$; clypeus width/height: $3.5 \times$. Length/width of flagellomeres: 2nd (2.4x), 8th (2.5x), 14th (1.3x). Length of flagellomere $2^{\text {nd }} /$ length of flagellomere $14^{\text {th }}: 2.2 \times$. Ocello-ocular distance/posterior ocelli diameter: $2.2 \times$; distance between posterior ocelli/ocelli diameter: $1.3 \times$.

Mesosoma. Pronotum with two lateral grooves present, the lower one excavated. Mesoscutum more or less uniformly sculptured by shallowly impressed punctures (distance between punctures about the same as their diameter). Mesoscutum $1.3 \times$ wider than long. Mesoscutum and scutellum uniformly covered by dense, pale yellow pilosity. Scutellum mostly smooth, with very shallow and sparse punctures. Scutellum length/width at base 1.1×. Scutellar suture broad, with 4-6 costulae. Posterior band of scutellum polished. Scutellar lateral face with polished area less than $20 \%$ the face height and less than half the face width. Mesopleuron mostly smooth and glabrous, except for punctures on the anterior margin and setae on all margins; separated from metapleuron by crenulate sulcus. Metapleuron mostly smooth, with some punctures and setae in the apical half; metapleuron with a crenulated, longitudinal sulcus running from lower margin near metacoxa through spiracle. Metapleural carina raised with a short lamella. Propodeum mostly smooth; with a median carina well defined and raised its entire length; and with a clearly complete transverse carina that reaches the spiracles and forks around them (there are also additional, shorter transverse carinae). Transverse carina on propodeum delimiting two areas, the anterior, basal one that is more or less horizontal, and the posterior, apical one is declivous.

Metasoma. Mediotergite 1 mostly smooth and with a deep medial groove over its basal half; slightly widening for the first quarter of its length, then narrowing to- 

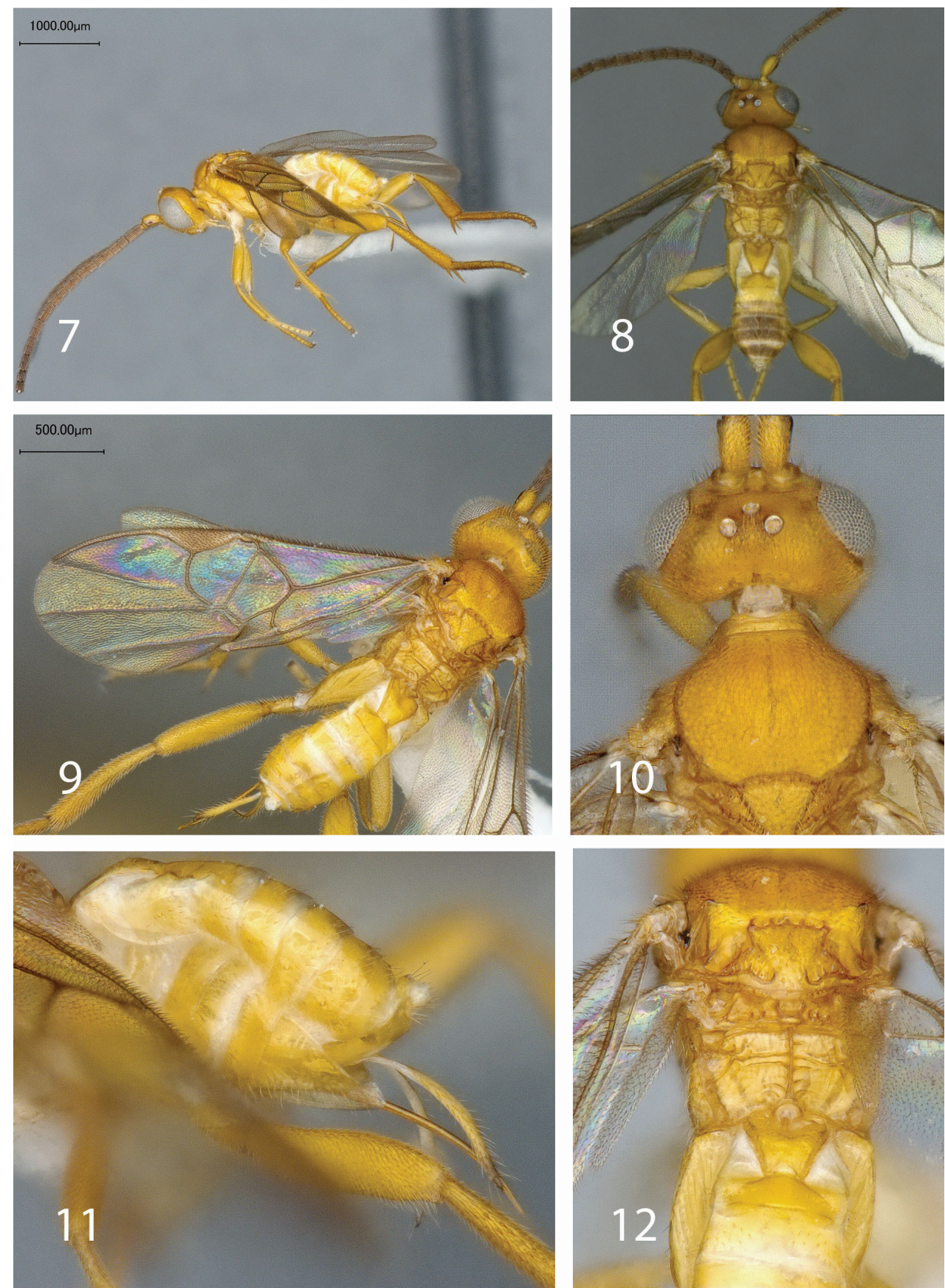

Figures 7-12. Mariapanteles dapkeyae Fernandez-Triana. 7 lateral habitus, female 8 dorsal habitus, female $\mathbf{9}$ fore wing, female $\mathbf{I} \mathbf{0}$ head and mesoscutum, dorsal view, female II hypopygium and ovipositor, lateral view $\mathbf{I} \mathbf{2}$ metanotum, propodeum and anterior metasomal tergites, female, dorsal view. 
wards apex; basal width/apical width $1.5 \times$; length/apical width $3.3 \times$. Mediotergite 2 mostly smooth, transverse, subtriangular to trapezoidal in shape; basal width/apical width $0.3 \times$; length/apical width $0.4 \times$. Mediotergite $31.5 \times$ the length of mediotergite 2. Mediotergite 3 and following unsculptured, polished and with sparse setae. Hypopygium mostly inflexible but with a median, translucid fold ventrally where 1-2 weak pleats are sometimes distinguishable. Ovipositor sheaths fully setose, $0.7 \times$ as long as metatibia length.

Legs. Metacoxa long, surpassing the length of the third metasomal tergum. Metatibial inner spur $1.4 \times$ the length of outer spur, and $0.5 \mathrm{X}$ the length of metatarsomere 1. Metafemur $3.2 \times$ as long as wide.

Wings. Vein R1a $1.3 \times$ as long as stigma length. Stigma $3.3 \times$ as long as wide. Length of R1a about $14 \times$ as long as the distance between its end and the end of $3 \mathrm{RSb}$. Vein $r$ and 2RS evenly curved to very slightly arched, with no clear limits between the two veins. Vein $2 \mathrm{M}$ about the same length of vein $(\mathrm{RS}+\mathrm{M}) \mathrm{b}$. Edge of vannal lobe of hind wing medially straight to slightly concave and with uniformly distribute setae which are shorter than those at base and apex of the lobe.

Colour: Mostly yellow, with antennal flagellomere, forewing stigma and most of the wing veins, light brown.

Male. Mostly like females, but some specimens with darker interocellar area and mediotergites $3+$. We associate these males with these females because of their morphological similarity.

Distribution. The specimens were collected with Malaise traps in two Brazilian localities (less than $1000 \mathrm{~km}$ apart) which are presumed to have been rain forests at the time of collecting.

Molecular data. Two paratype male specimens rendered partial barcodes (361 bp for the one with DNA voucher code CNCHYM 03387, and $164 \mathrm{bp}$ for the one with DNA voucher code CNCHYM 07145). The nucleotide sequence shown below corresponds to the longer sequence in the barcode region of COI:

$>$ Mariapanteles dapkeyae

TAAGATTTTGATTATTAATTCCATCTTTATTTATATTAATTTTT AGAAGATTTATTAATACAGGAGTAGGTACAGGTTGAACAGTATACCCACCATTATCATCAAATTTAAGACATAGGGGCATATCAGTCGATT TAAGAATTTTTTCTTTACATTTAGCAGGAACTTCATCAATTATAGGAGCAATTAATTTTATTACAACAATTAAAAATATACGAGTTAAAT TATTTAAAATAAATAAAATTTCTTTATTTAATTGATCAGTTTTAATTACAGCAATTTTATTATTATTATCATTACCAGTATTAGCAGGTGCTATTACTATACTTTTAACAGATCGAAATTTAAATACATCATTTTT

Etymology. This species is dedicated to Tanya Heckmann Dapkey of Philadelphia, Pennsylvania, USA, in recognition of her seven years of diligent and highly accurate sorting, processing, databasing, and de-legging ACG microgastrine wasps for DNA barcoding.

Comments. The biology of this species, collected with Malaise traps, is unknown. In the $\mathrm{CNC}$ collection there are two additional specimens of Mariapanteles from Bra- 


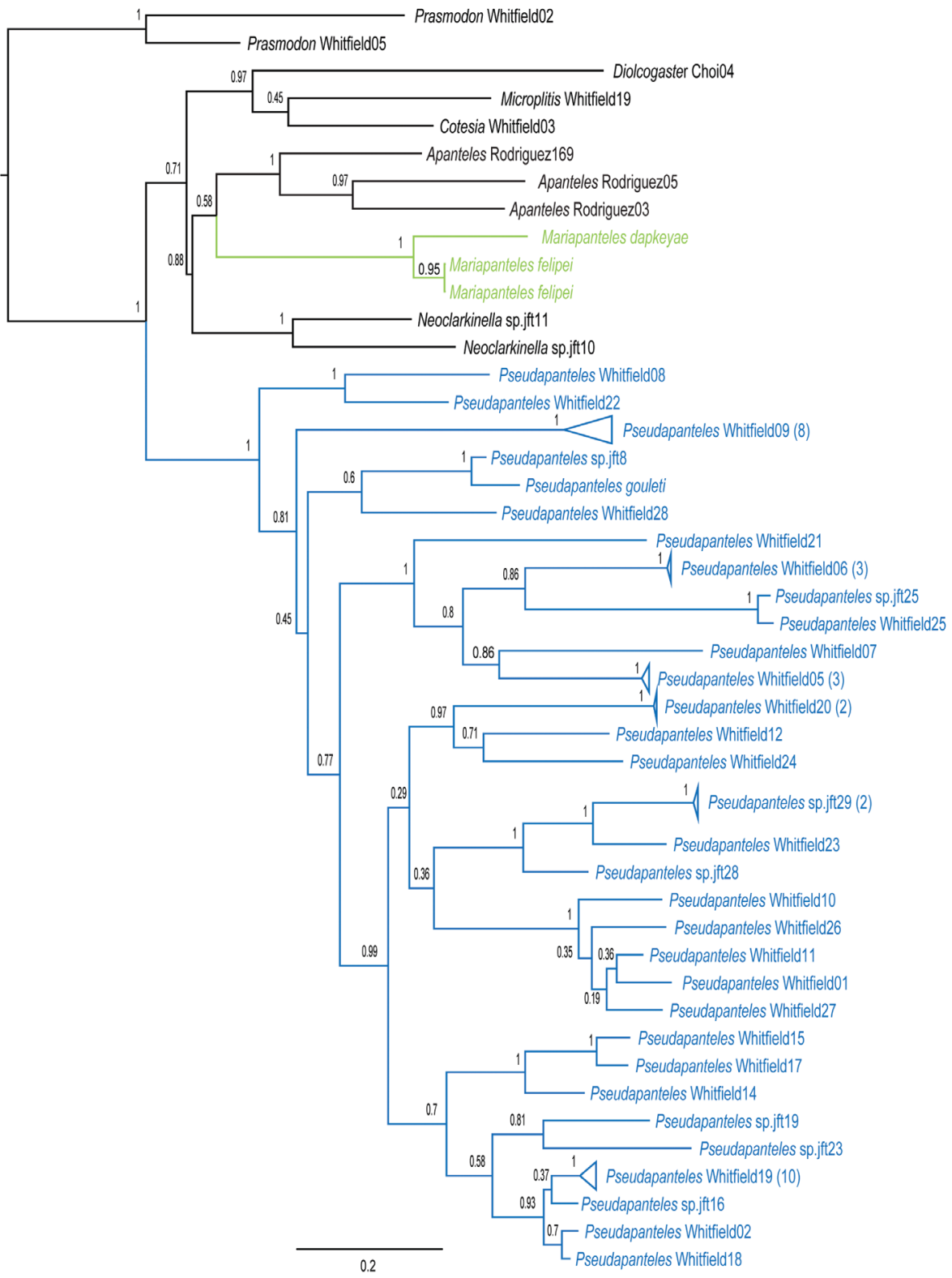

Figure 13. Maximum clade credibility tree for Pseudapanteles and Mariapanteles based on Bayesian analysis of COI sequences, with $3^{\text {rd }}$ codon positions included (see text for details). Values at nodes are posterior probabilities.

zil: one female from Piedra Azul, Minas Gerais; and one male from Rio Javari, Estirar do Equador, Amazonas. Both specimens differ morphologically from M. dapkeyae. Additionally, the male specimen (with DNA voucher code CNCHYM 03380) rendered a partial DNA barcode (164bp) which has 7 base pairs different (4.3\%) from 


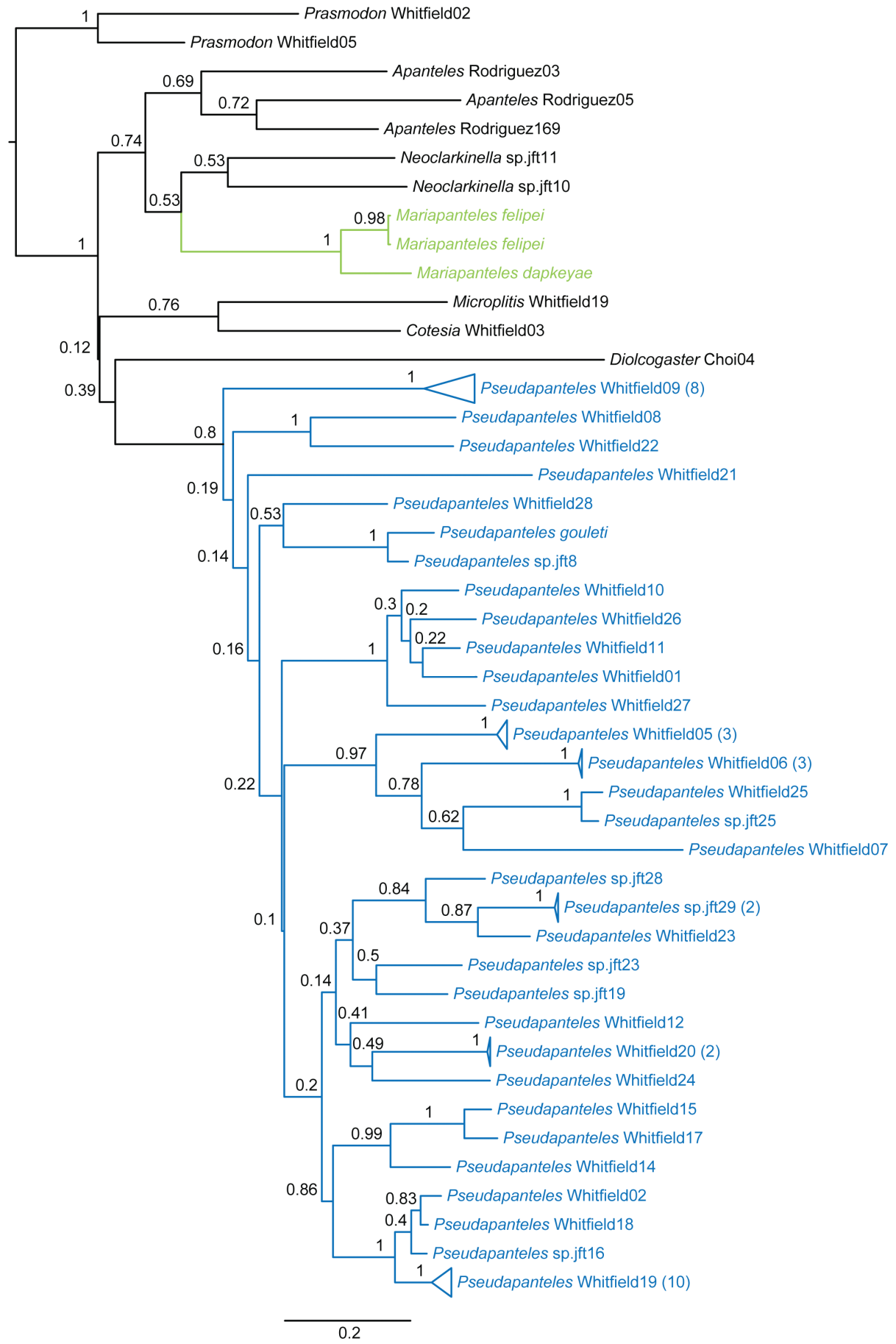

Figure 14. Maximum clade credibility tree for Pseudapanteles and Mariapanteles based on Bayesian analysis of COI sequences, with $3^{\text {rd }}$ codon positions excluded (see text for details). Values at nodes are posterior probabilities. 
the barcoded specimens of $M$. dapkeyae. We believe those two specimens may represent additional species, but because they are singletons we have not described them as new species here.

\section{Acknowledgments}

We emphatically and gratefully acknowledge the support of the ACG parataxonomist team in finding and rearing the caterpillars, their parasitoids and their hyperparasitoids, and Area de Conservacion Guanacaste (ACG) for preserving the forests in which they live, and the Guanacaste Dry Forest Conservation Fund, the Wege Foundation, the International Conservation Fund of Canada, the JRS Biodiversity Foundation, Jessie Hill, and the University of Pennsylvania for funding portions of the research. This study was also supported by NSF DEB 0515699 to DHJ and by a Natural Sciences and Engineering Research Council of Canada (NSERC) Discovery Grant to MAS. Laboratory analyses of these sequences were funded by the Government of Canada through Genome Canada and the Ontario Genomics Institute (2008-0GI-ICI-03).

\section{References}

Burns JM, Janzen DH, Hajibabaei M, Hallwachs W, Hebert PDN (2007) DNA barcodes of closely related (but morphologically and ecologically distinct) species of skipper butterflies (Hesperiidae) can differ by only one to three nucleotides. Journal of the Lepidopterists' Society 61(3): 138-153.

Burns JM, Janzen DH, Hajibabaei M, Hallwachs W, Hebert PDN (2008) DNA barcodes and cryptic species of skipper butterflies in the genus Perichares in Area de Conservación Guanacaste, Costa Rica. Proceedings of the National Academy of Sciences 105: 6350-6355. doi: $10.1073 /$ pnas.0712181105

Campos M, Diego F (2001) Lista de los generos de avispas parasitoides Braconidae (Hymenoptera: Ichneumonoidea) de la region neotropical. Biota Colombiana 2(3): 193-232.

Deans AR, Whitfield JB, Janzen DH (2003) Taxonomy and natural history of the microgastrine genus Alphomelon Mason (Hymenoptera: Braconidae). Journal of Hymenoptera Research 12(1): 1-41.

Drummond AJ, Ashton B, Buxton S, Cheung M, Cooper A, Duran C, Field M, Heled J, Kearse M, Markowitz S, Moir R, Stones-Havas S, Sturrock S, Thierer T, Wilson A (2012) Geneious v5.5.6, http://www.geneious.com/

Gauld ID, Janzen DH (2004) The systematics and biology of the Costa Rican species of parasitic wasps in the Thyreodon genus-group (Hymenoptera: Ichneumonidae). Zoological Journal of the Linnean Society 141: 297-351. doi: 10.1111/j.1096-3642.2004.00116.x

Grinter CC, Whitfield, JB, Connahs, H, Dyer LA, Hallwachs W, Janzen DH (2009) A key to New World Distatrix Mason (Hymenoptera: Braconidae), with descriptions of six new reared Neotropical species. Journal of Insect Science 9(25):1-15. doi: 10.1673/031.009.2901 
Ivanova NV, Dewaard JR, Hebert PDN (2006) An inexpensive, automation-friendly protocol for recovering high-quality DNA. Molecular Ecology Notes 6: 998-1002. doi: 10.1111/j.1471-8286.2006.01428.x

Janzen DH (2000) Costa Rica's Area de Conservación Guanacaste: a long march to survival through non-damaging biodevelopment. Biodiversity 1(2): 7-20. doi: 10.1080/14888386.2000.9712501

Janzen DH, Hallwachs W (2011) Joining inventory by parataxonomists with DNA barcoding of a large complex tropical conserved wildland in northwestern Costa Rica. PLoS ONE 6(8): e18123. doi: 10.1371/journal.pone.0018123

Janzen DH, Hallwachs W (2012) Philosophy, navigation and use of a dynamic database ("ACG Caterpillars SRNP”) for an inventory of the macrocaterpillar fauna, and its food plants and parasitoids, of the Area de Conservacion Guanacaste (ACG), northwestern Costa Rica http://janzen.sas.upenn.edu

Janzen DH, Hallwachs W, Blandin P, Burns JM, Cadiou J, Chacon I, Dapkey T, Deans AR, Epstein ME, Espinoza B, Franclemont JG, Haber WA, Hajibabaei M, Hall JPW, Hebert PDN, Gauld ID, Harvey DJ, Hausmann A, Kitching I, Lafontaine D, Landry J, Lemaire C, Miller JY, Miller JS, Miller L, Miller SE, Montero J, Munroe E, Rab Green S, Ratnasingham S, Rawlins JE, Robbins RK, Rodriguez JJ, Rougerie R, Sharkey MJ, Smith MA, Solis MA, Sullivan JB, Thiaucourt P, Wahl DB, Weller SJ, Whitfield JB, Willmott KR, Wood DM, Woodley NE, Wilson JJ (2009) Integration of DNA barcoding into an ongoing inventory of complex tropical biodiversity. Molecular Ecology Resources 9 (Supplement 1): 1-26. doi: 10.1111/j.1755-0998.2009.02628.x

Janzen DH, Hallwachs W, Burns JM, Hajibabaei M, Bertrand C, Hebert PDN (2011) Reading the complex skipper fauna of one tropical place. PLoS ONE 6(8): e19874. doi: 10.1371/ journal.pone.0019874

Miller MA, Pfeiffer W, Schwartz T (2010) "Creating the CIPRES Science Gateway for inference of large phylogenetic trees" in Proceedings of the Gateway Computing Environments Workshop (GCE), 14 Nov. 2010, New Orleans, LA 1-8.

Posada D (2008) jModelTest: Phylogenetic model averaging. Molecular Biology and Evolution 25: 1253. doi: $10.1093 / \mathrm{molbev} / \mathrm{msn} 083$

Rambaut A, Drummond AJ (2009) Tracer v1.5, Available from http://beast.bio.ed.ac.uk/Tracer Rambaut A, Drummond AJ (2012) TreeAnnotator v1.7.0, Available from http://beast.bio. ed.ac.uk

Ratnasingham S, Hebert PDN (2007) BOLD : The Barcode of Life Data System (www. barcodinglife.org). Molecular Ecology Notes 7: 355-364. doi: 10.1111/j.14718286.2007.01678.x

Ronquist F, Huelsenbeck JP (2003) MRBAYES 3: Bayesian phylogenetic inference under mixed models. Bioinformatics 19: 1572-1574. doi: 10.1093/bioinformatics/btg180

Schauff ME, Janzen DH (2001) Taxonomy and ecology of Costa Rican Euplectrus (Hymenoptera: Eulophidae), parasitoids of caterpillars (Lepidoptera). Journal of Hymenoptera Research 10(2): 181-230.

Sharkey MJ, Clutts S, Tucker EM, Janzen DH, Hallwachs W, Dapkey T, Smith MA (2011) Lytopylus Forster (Hymenoptera, Braconidae, Agathidinae) species from Costa Rica, with 
an emphasis on specimens reared from caterpillars in Area de Conservacion Guanacaste. In: Shcherbakov DE, Engel MS, Sharkey MJ (Eds) Advances in the Systematics of Fossil and Modern Insects: Honouring Alexandr Rasnitsyn. ZooKeys 130: 379-419. doi: 10.3897/zookeys.130.1569

Smith MA, Rodriguez JJ, Whitfield JB, Janzen DH, Hallwachs W, Deans AR, Hebert PDN (2008) Extreme diversity of tropical parasitoid wasps exposed by iterative integration of natural history, DNA barcoding, morphology and collections. Proceedings of the National Academy of Sciences of the USA 105: 12359-12364. doi: 10.1073/pnas.0805319105

Smith MA, Wood DM, Janzen DH, Hallwachs W, Hebert PDN (2007) DNA barcodes affirm that 16 species of apparently generalist tropical parasitoid flies (Diptera, Tachinidae) are not all generalists. Proceedings of the National Academy of Sciences 104: 4967-4972. doi: 10.1073/pnas.0700050104

Smith MA, Woodley NE, Janzen DH, Hallwachs W, Hebert PDN (2006) DNA barcodes reveal cryptic host-specificity within the presumed polyphagous members of a genus of parasitoid flies (Diptera: Tachinidae). Proceedings of the National Academy of Sciences 103: 3657-3662. doi: 10.1073/pnas.0511318103

Valerio AA, Whitfield JB, Janzen DH (2009) Review of world Parapanteles Ashmead (Hymenoptera: Braconidae: Microgastrinae), with descriptions of fourteen new Neotropical species and the first description of the final instar larvae. Zootaxa 2084: 1-49.

Whitfield JB, Rasmussen C, Arias Penna DC (2011) Review of the New World microgastrine braconid wasp genus Venanus Mason, with descriptions of three new Neotropical species. Annals of the Entomological Society of America 104: 1119-1127. doi: 10.1603/AN10048 


\section{Appendix I}

Detailed information about collection, BOLD, and GenBank number of species. (doi: 10.3897/zookeys.208.3326.app) File format: Excel spreadsheet (xls).

Explanation note: Appendix I contains all available DNA barcodes for Pseudapanteles (32 New World species, most of which are undescribed) and Mariapanteles (2 species) that were at least $300 \mathrm{bp}$ long were downloaded from BOLD.

Copyright notice: This dataset is made available under the Open Database License (http://opendatacommons.org/licenses/odbl/1.0/). The Open Database License (ODbL) is a license agreement intended to allow users to freely share, modify, and use this Dataset while maintaining this same freedom for others, provided that the original source and author(s) are credited.

Citation: Whitfield JB, Fernández-Triana JL, Janzen DH, Hallwachs W, Smith MA, Cardinal S (2012) Mariapanteles (Hymenoptera, Braconidae), a new genus of Neotropical microgastrine parasitoid wasp discovered through biodiversity inventory. ZooKeys 208: 61-80. doi: 10.3897/zookeys.208.3326.app 